Published in final edited form as:

J Am Acad Dermatol. 2018 March ; 78(3): 607-609. doi:10.1016/j.jaad.2017.08.038.

\title{
The microbial flora of taxane therapy-associated nail disease in cancer patients
}

\author{
Cesar A. Virgen, MD, PhD ${ }^{1,2}$, Viswanath R. Belum, MD $^{3}$, Mini Kamboj, MD ${ }^{4}$, Shari B. \\ Goldfarb, $\mathbf{M D}^{5}$, Victoria S. Blinder, $\mathbf{M D}^{6}$, Ayca Gucalp, $\mathbf{M D}^{5}$, and Mario E. Lacouture, MD $^{3}$ \\ ${ }^{1}$ Department of Medicine, University of California San Diego, La Jolla, CA \\ ${ }^{3}$ Dermatology Service, Memorial Sloan Kettering Cancer Center, New York, NY \\ ${ }^{4}$ Infectious Diseases Service, Memorial Sloan Kettering Cancer Center, New York, NY \\ ${ }^{5}$ Breast Medicine Service, Memorial Sloan Kettering Cancer Center, New York, NY \\ ${ }^{6}$ Epidemiology and Biostatistics, Memorial Sloan Kettering Cancer Center, New York, NY
}

\section{Keywords}

taxane; paclitaxel; docetaxel; paronychia; onycholysis; onychodystrophy; microbial flora; microbiota

\section{TO THE EDITOR}

Taxanes are frequently associated with nail and periungual tissue afflictions such as paronychia, onychodystrophy, onycholysis, subungual hemorrhages, and pyogenic granulomas. ${ }^{1}$ These therapy-associated nail abnormalities can cause physical symptoms and psychosocial distress, leading to impairments in patients' quality of life (QoL). The changes in QoL may impact the number of therapy cycles that a patient can tolerate, and in some cases, they may lead to discontinuation of anti-cancer therapy. ${ }^{2}$

\footnotetext{
Correspondence: Mario E. Lacouture, MD, Dermatology Service, Department of Medicine, Memorial Sloan Kettering Cancer Center, 60th Street Outpatient Center, 16 East 60th St., Suite 407, Room 4312, New York, NY 10022, Phone: (646) 888-6014 | Fax: (646) 227-7274, LacoutuM@mskcc.org.

${ }^{2}$ Current address: Department of Dermatology, University of California Irvine, Irvine, CA

Conflict of Interest disclosures: None reported.

Statement on prior presentations: The contents of this manuscript have not been previously published and are not currently submitted elsewhere.

IRB Review: The study was approved by the Institutional Review Board of Memorial Sloan Kettering Cancer Center.

Financial Disclosure: CAV, VRB, and MK have no relevant financial affiliations. SBG receives research funding from Berg Health and HealthTell, and she is an advisory board member and consultant for Sermonix Pharmaceuticals. VSB is a consultant for Anthem Foundation, Pfizer, and US Business Leadership Network. AG receives research funding from Innocrin Pharmaceuticals and Pfizer. MEL reports that he is serving as a consultant to Quintiles, Boehringer Ingelheim, Genentech, Foamix, Infinity Pharmaceuticals, Janssen R\&D, Novartis, and AstraZeneca pharmaceuticals. He has received research funding from Bristol-Myers Squibb, and Berg.

Publisher's Disclaimer: This is a PDF file of an unedited manuscript that has been accepted for publication. As a service to our customers we are providing this early version of the manuscript. The manuscript will undergo copyediting, typesetting, and review of the resulting proof before it is published in its final citable form. Please note that during the production process errors may be discovered which could affect the content, and all legal disclaimers that apply to the journal pertain.
} 
We conducted a retrospective chart review (8/2012-6/2015) of paclitaxel and docetaxeltreated patients referred to a dermatologist (M.E.L.). Of 114 patients, a majority were women with breast cancer, and had developed paclitaxel-associated acute paronychia (Table I). The clinical severity of the nail infections and changes was mostly grade 2 , consistent with moderate severity adverse events (AEs) requiring local, noninvasive interventions (CTCAE $\mathrm{v} 4.0^{3}$; Table I). We did not identify nail abnormalities with grade 3 or 4 , suggesting that medically significant AEs may require prompt interruption of anticancer therapy and are therefore less likely to be seen in the outpatient setting.

Microbiological cultures grew 21 distinct bacterial and fungal species (Figure 1). The most commonly isolated organism, Staphylococcus aureus, is a part of resident skin flora. In cancer patients however, it is associated with a high morbidity and mortality rate. ${ }^{4}$ Nearly half of the isolates in these patients were opportunistic and/or pathogenic (non-skin flora) organisms. This is reflective of the combined role of impaired resistance to colonization because of taxane therapy and exposure to healthcare environments where the acquisition of these organisms is more likely. In general, direct cytotoxic, antiangiogenic, or neurotropic effects of taxanes in the nail matrix appear to be the inciting factors, with a positive correlation to the number of treatment cycles (cumulative dose). In the setting of an impaired physical barrier due to the antimitotic effect of taxanes in the nail matrix, and suppression of the innate and adaptive immune responses related to systemic cytotoxic anticancer therapies, we hypothesize that these infectious agents may be responsible for nail abnormalities in some patients.

In 46 patients (40.4\%), the culture sensitivity results necessitated treatment modifications. The most prescribed oral antibiotics were cefadroxil, ciprofloxacin, and sulfamethoxazoletrimethoprim. The most prescribed topical antimicrobials were ciclopirox, bacitracinpolymyxin $b$, and iodine. At follow-up, $80.5 \%$ of patients reported clinical improvement in their condition (Table I).

In the cancer patient population, we recommend routinely collecting bacterial and fungal culture swabs from affected periungual tissues. We propose empiric oral antibiotic treatment with fluoroquinolones or first- or second-generation cephalosporins in conjunction with topical antimicrobials. In the author's (M.E.L.) experience, extended therapy (4-6 weeks) is often required, perhaps due to poor penetration of antibiotics into affected tissues and risk of treatment failure with shorter courses ( $\leq 2$ weeks). Of note, nail infections and other nail changes are likely to recur if taxane therapy is ongoing. Instituting frozen gel sock and/or glove therapy for fifteen minutes before, sixty minutes during, and fifteen minutes after taxane infusion reduces the incidence of nail pathology. ${ }^{5}$ Patients will need routine supportive oncodermatology monitoring.

\section{Acknowledgments}

We would like to thank Kathryn Ciccolini, RN and Daphne Leon for assisting with the ePathbook database in the Dermatology Service, MSKCC.

Funding/Support: CAV was supported by the American Academy of Dermatology (AAD) Diversity Mentorship Program and the National Institutes of Health (NIH) Training Grant T32-GM007198. This study was partially 
supported by the NIH Cancer Center Support Grant P30-CA008748. VRB and MEL are supported by the RJR Oncodermatology Fund at Memorial Sloan Kettering Cancer Center.

\section{Abbreviations}

QoL

\section{References}

1. Sibaud V, Lebœuf NR, Roche H, et al. Dermatological adverse events with taxane chemotherapy. Eur J Dermatol. 2016; 26(5):427-443. [PubMed: 27550571]

2. Winther D, Saunte DM, Knap M, et al. Nail changes due to docetaxel--a neglected side effect and nuisance for the patient. Support Care Cancer. 2007; 15(10):1191-1197. [PubMed: 17318589]

3. Chen AP, Setser A, Anadkat MJ, et al. Grading dermatologic adverse events of cancer treatments: The Common Terminology Criteria for Adverse Events Version 4.0. J Am Acad Dermatol. 2012; 67(5):1025-1039. [PubMed: 22502948]

4. Gopal AK, Fowler VG Jr, Shah M, et al. Prospective analysis of Staphylococcus aureus bacteremia in nonneutropenic adults with malignancy. J Clin Oncol. 2000; 18(5):1110-1115. [PubMed: 10694564]

5. Scotté F, Banu E, Medioni J, et al. Matched case-control phase 2 study to evaluate the use of a frozen sock to prevent docetaxel-induced onycholysis and cutaneous toxicity of the foot. Cancer. 2008; 112(7):1625-31. [PubMed: 18286527] 


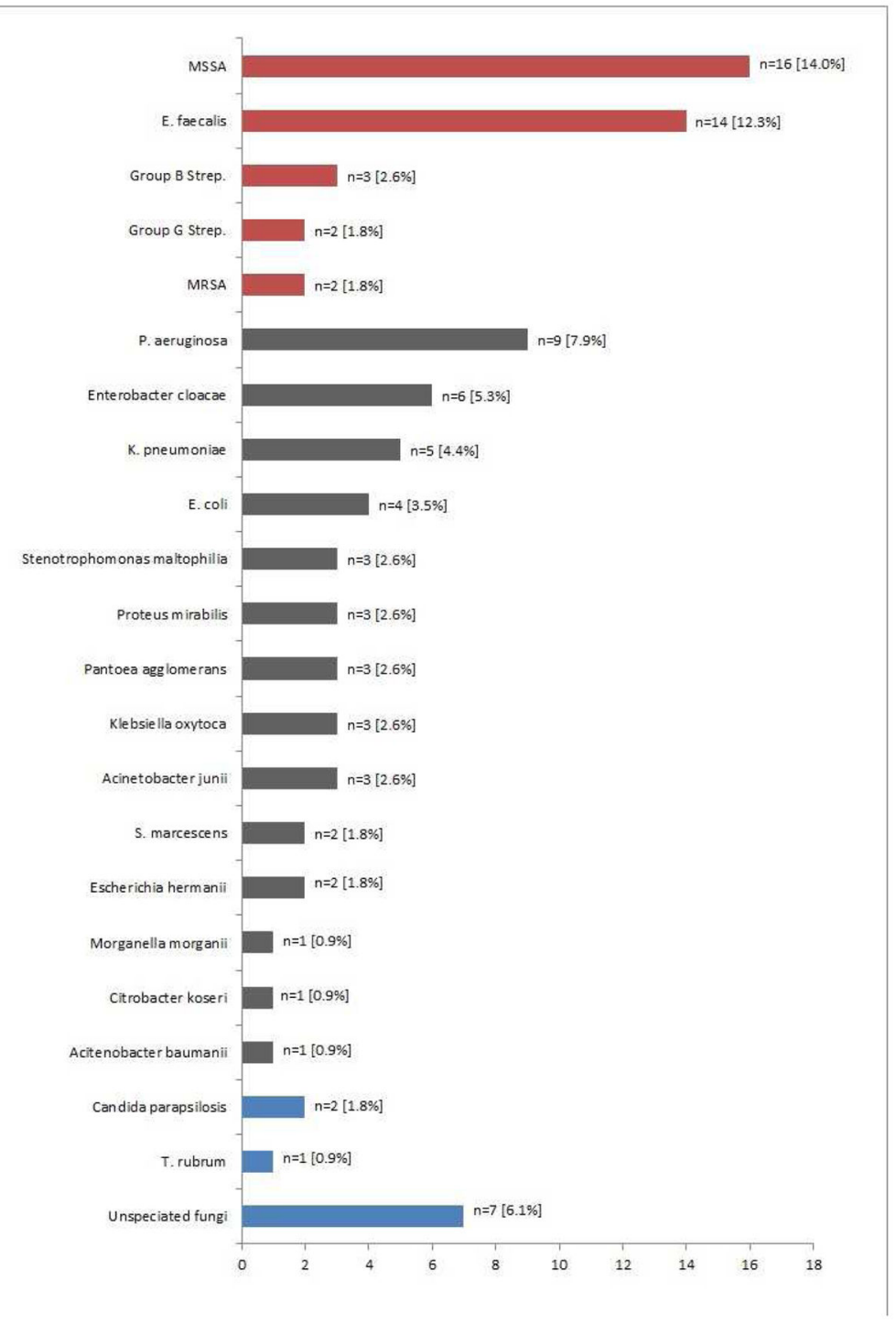

Figure 1.

Gram-positive bacteria (red bars), Gram-negative bacteria (gray bars), and fungi (blue bars) isolated from affected tissues in patients with taxane-associated nail infections and changes. 


\section{Table I}

Characteristics of patients with taxane-associated nail infections and nail changes.

\begin{tabular}{lll}
\hline A. Descriptive summary of demographic characteristics of the study sample (n=114). \\
\hline Feature & No. & $(\%)$ \\
\hline Gender & 11 & $(9.6)$ \\
Male & 103 & $(90.4)$ \\
Female & 55.2 & \\
Mean age & & \\
Cancer type or site & 84 & $(73.7)$ \\
Breast & 10 & $(8.8)$ \\
Lung & 10 & $(8.8)$ \\
Gastrointestinal & 7 & $(6.1)$ \\
Gynecologic & 3 & $(2.6)$ \\
Other & & \\
Cancer treatment & 95 & $(83.3)$ \\
Paclitaxel & 15 & $(3.5)$ \\
Docetaxel & 4 & \\
Paclitaxel and docetaxel &
\end{tabular}

B. Clinical severity grading of taxane-associated nail infections and nail changes.

\begin{tabular}{lcccc}
\hline & \multicolumn{2}{c}{ Nail infection } & \multicolumn{2}{c}{ Nail changes } \\
& No. & $(\%)$ & No. & $(\%)$ \\
\hline Grading noted & & & & \\
Grade 1 & 9 & $(11.7)$ & 24 & $(26.1)$ \\
Grade 2 & 68 & $(88.3)$ & 68 & $(73.9)$ \\
Grade 3 & 0 & $(0.0)$ & 0 & $(0.0)$ \\
Grade 4 & 0 & $(0.0)$ & 0 & $(0.0)$ \\
Grading not noted & 4 & & 5 & \\
\hline
\end{tabular}

C. Treatment instituted for taxane-associated nail infections and nail changes.

\begin{tabular}{lcc}
\hline Feature & No. & $(\%)$ \\
\hline Antimicrobial therapy (n=114) & 37 & $(32.5)$ \\
$\quad$ Topical agents alone & 0 & $(0.0)$ \\
$\quad$ Oral agents alone & 77 & $(67.5)$ \\
$\quad$ Topical and oral combinations & 66 & $(80.5)$ \\
Nail symptoms improved (n=82) & 16 & $(19.5)$ \\
$\quad$ Yes & 32 & \\
$\quad$ No & & \\
No dermatology follow-up &
\end{tabular}

